\title{
O Controle de Infecção Hospitalar como indicador para Qualidade no Serviço de Saúde
}

Michelly de Melo Alves ${ }^{1}$; Geraldo Sadoyama Leal'; Adriana dos Santos Prado Sadoyama3; Ednólia Gomes Varjão Fernandes" ${ }^{4}$ : Mabel Duarte Alves Gomide ${ }^{5}$, Amanda Oliveira Soares Monteiro Silveira6; Reila Silva Pereira Aires ${ }^{7}$

\section{Resumo}

A qualidade dos serviços está diretamente relacionada à capacidade de satisfazer as necessidades dos clientes. O movimento pela qualidade têm se expandido para o setor da saúde e o controle de infecção hospitalar é uma das medidas para avaliação da qualidade da assistência, pois tal controle permite que a instituição de saúde avalie a estrutura em que o serviço é prestado, como está sendo realizado e os resultados dessa assistência, evidenciado pelo aumento ou diminuição das infecções relacionadas à saúde. A avaliação da prática de higienização das mãos dos profissionais de saúde é uma das medidas mais importantes para verificar a aderência à prática pelos profissionais, pois, as IRAS são uma grande maioria das causas de morbimortalidade em pessoas que se submetem a procedimentos

\footnotetext{
1 Enfermeira - Professora do Curso de enfermagem - UAE de Biotecnologia; Mestranda do Mestrado Profissional em Gestão Organizacional/RC/UFG. E-mail: michellymeloa@hotmail.com

2 Biólogo - UAE de Biotecnologia; Professor do Mestrado Profissional em Gestão Organizacional/RC/UFG. E-mail: gsadoyama@gmail.com

3 Linguista e Pedagoga - UAE de Educação; Professora do Mestrado Profissional em Gestão Organizacional/RC/UFG. Email:drisadoyama@gmail.com

4 Enfermeira - Hospital São Nicolau. Email- ednoliavarjao@yahoo.com.br

5 Médica - Dermatologista. Mestre em Gestão Organizacional UFG/RC. Email: Mabel@ dermaclinicagoias.com.br

6 Médica - Endocrinologista. Mestranda em Gestão Organizacional UFG/RC. Email: Amanda.osm@bol.com.br

7 Médica - Intensivista. Mestranda em Gestão OrganizacionalUFG/RC. Email: reilap@gmail.com
} 
clínicos, e a mão dos profissionais de saúde constituem-se na principal via de transmissão de microorganismos.

Palavras-chave: infecção hospitalar; controle de qualidade; cultura organizacional

\section{INTRODUÇÃO}

Atualmente a qualidade dos serviços principalmente em organizações na qual se têm uma grande quantidade de clientes é uma constante preocupação. A falta de qualidade se deve ao fato de que algumas instituições não se preocupam em avaliar o grau de satisfação de seus clientes ou até mesmo não sabem como avaliá-la (ASEFZADEH et al. 2016).

O controle de infecção hospitalar é uma das medidas para avaliação da qualidade da assistência em instituições Hospitalares. As atividades desempenhadas pela equipe de controle de infecção hospitalar adotam estratégias para melhoria na qualidade do serviço e quando realizadas corretamente permite a promoção de medidas eficazes para prevenção de infecções hospitalares, visto que, esta atividade na instituição de saúde tem como objetivo avaliar a estrutura em que o serviço é prestado, como está sendo realizado e os resultados dessa assistência, evidenciado pelo aumento ou diminuição das infecções relacionadas à saúde (DUTRA, COSTA et al. 2015).

As Infecções Relacionadas à Assistência à Saúde (IRAS) são consideradas como uma grande maioria das causas de morbimortalidade em pessoas que se submetem a procedimentos clínicos. A realidade é que muitas vezes a prática de controle de infecção hospitalar não é aderida por uma grande maioria dos profissionais de saúde, havendo dessa forma, uma necessidade de se investigar porque esses profissionais têm essa dificuldade de adesão e programar estratégias para que esse controle de infecção seja eficaz, proporcionando a segurança do paciente e melhorar a qualidade do serviço (SOUZA, BELEl et al. 2015).

\section{DESENVOLVIMENTO}

\subsection{A Qualidade nos Serviços de Saúde}

$\mathrm{Na}$ área da saúde a busca pelo sistema de qualidade é cada vez crescente, na qual mudanças significativas têm sido notadas no Sistema Único de Saúde (SUS) e nos modelos de gestão das unidades de saúde (ALBUQUERQUE, COSTA e SALAZAR, 2012). 
$\mathrm{Na}$ área da saúde há uma grande exigência pela busca da qualidade, além disso, é importante que os profissionais tenham conhecimento da realidade do serviço de saúde que é oferecido à população analisando pontos positivos e negativos que promovam reflexão e adoção de medidas para melhoria na qualidade da assistência. Esta avaliação do sistema de qualidade é proposta pelo sistema Gestão da Qualidade (GQ) que surgiu em meados do século XX e que tem disseminado na área da saúde (PADILHA e MATSUDA, 2011).

A prática dos profissionais de saúde nas instituições hospitalares é classificada como fator de influência na qualidade e segurança da assistência prestada ao paciente. $O$ ambiente hospitalar que se encontra com melhores condições de trabalho a prática assistencial, a segurança do paciente e a qualidade do cuidado acontecem de forma mais adequada, diminuindo a ocorrência de eventos adversos (ALVES e GUIRARDELLO, 2016)

Um dos grandes desafios para os gestores dentro de uma instituição consiste na cultura de segurança que está inserida no ambiente de trabalho. Fatores como a satisfação no trabalho, a forma com que os profissionais da instituição lidam com erros e eventos adversos que venham a ocorrer e a percepção dos profissionais em relação à gestão são indicadores que permite avaliar as atitudes que favorecem a cultura de segurança nas instituições (MASSOCO e MELLEIRO, 2015).

Para que um cuidado à saúde se torne seguro é fundamental que se construa esta cultura institucional dentro da organização, no qual, o Programa Nacional de Segurança do Paciente (PNSP) a define como (URBANETTO e GERHARDT, 2013):

- Cultura no qual todos os profissionais e funcionários da instituição assumam responsabilidades pela sua segurança, de seus colegas, dos pacientes e familiares

- Cultura no qual priorizará a segurança acima de questões financeiras

- Cultura que encoraja e recompensa a identificação, notificação e resolução de problemas

- Cultura que a partir da ocorrência de algum evento adverso promova o aprendizado e capacitação profissional

- Cultura que proporciona recursos e estrutura que viabiliza a realização efetiva da segurança 
A qualidade no cuidado na área da saúde é uma preocupação principalmente na enfermagem, no qual é necessária uma busca contínua pela qualidade, por meio de uma avaliação contínua da assistência seguida por um planejamento estratégico, controle e redução de custos (OLIVEIRA et al. 2015).

\subsection{Gestão da Qualidade}

Com a inserção da Gestão da Qualidade Total ou Gerenciamento da Qualidade Total (QGT) como um novo modelo de gerenciamento nas organizações de saúde os gestores passaram a ter como foco primordial o sistema de qualidade para se obter um diferencial na assistência prestada, que passa agora a influenciar na cultura organizacional da instituição que exige mudanças de atitude e comportamento dos profissionais, como comprometimento com as atividades assistenciais e aprimoramento do serviço. Contudo, a adoção da QGT nas instituições de saúde não foi implantada somente por uma exigência dos gestores dos serviços, mais também, por influência por parte do governo, clientes e da medicina (VITURI e ÉVORA, 2015).

As instituições de saúde para atingirem o padrão de qualidade muitas vezes adotam estratégias e modelos para alcançarem as metas propostas, que sem a utilização de instrumentos talvez não fosse possível alcançar os objetivos de acreditação e qualidade. Alguns modelos utilizados pelas instituições que trouxeram resultados positivos são (SCHIESARI, 2014):

- Aprimoramento da gestão: no qual o planejamento estratégico é o principal instrumento para a gestão, instrumento este que propicia a utilização de dados quantitativos para divulgação de indicadores que orientem as tomadas de decisões.

- Padronização dos processos: a elaboração de normas, rotinas, protocolos, documentos de orientação com a intenção de padronizar as ações.

- Treinamento do Pessoal: capacitação do profissional, incentivo a participação na educação permanente, com adequado planejamento das ações educativas.

- Trabalho em equipe: a atuação da equipe multiprofissional para assistência ao paciente constitui-se de grande importância para a qualidade no serviço prestado. 
- Cuidado Focado no Paciente: as instituições de saúde devem estar sempre atentas as necessidades dos pacientes para o planejamento dos cuidados ao indivíduo.

- Motivação: trabalhar a motivação dos funcionários para a busca da qualidade é caracterizada como medida fundamental para um crescimento no número de profissionais envolvidos nesse processo de melhoria da qualidade do serviço.

- Mudança da Cultura: a cultura organizacional é considerada de suma importância dentro de uma organização para a adequada realização dos processos do sistema de qualidade, no entanto, mudar o comportamento das pessoas utilizando os modelos citados acima em um primeiro momento pode ser fácil, porém, continuar no mesmo ritmo pode ser tarefa árdua e que requer uma mudança em longo prazo.

Os modelos adotados por instituições de saúde citados são de grande importância, mas, os gestores de cada instituição deverão analisar e se necessário elaborar outros modelos que sejam eficientes no processo de melhoria dos serviços prestados. As dificuldades para se progredir dentro do sistema de qualidade são inúmeras e que requerem a busca de métodos eficazes para sua realização juntamente com um bom desempenho por parte da gestão e os demais profissionais da instituição (SCHIESARI, 2014).

\subsection{Avaliação da Qualidade}

A escala SERVQUAL, desenvolvida por Parasuraman, Zithmal e Berry em meados dos anos 80 consiste em um dos melhores métodos utilizados para avaliar a qualidade do serviço nas perspectivas dos clientes, esta escala é de grande importância, pois, demonstra a diferença entre as percepções e expectativas, ou seja, realiza o levantamento da realidade vivenciada pelo cliente e os aspectos que necessitariam ser melhorados dentro da instituição (OLIAEE, JABBARI e EHSANPOUR, 2016). A escala avaliará a qualidade funcional do serviço, ou seja, a forma como o serviço é oferecido ao cliente e como muitas vezes os pacientes são incapazes de avaliar a qualidade técnica que consiste na avaliação de procedimentos médicos, a realização de uma análise da qualidade funcional tornase ponto-chave para se obter o levantamento da percepção do cliente sobre este serviço (BABAKUS e MANGOLD et al. 1992) 
Esta escala compreende 22 itens compostos por 5 dimensões sendo eles: tangibilidade, credibilidade, presteza, segurança e empatia (AGHAMOLAEI et al 2014).

Para Parasuraman et al. (1998) as 5 dimensões são compreendidas da seguinte forma:

- Tangibilidade: está relacionada com os equipamentos, infra-estrutura e aparência física.

- Credibilidade: habilidade e capacidade em realizar o serviço.

- Responsividade: se refere ao bom atendimento ao cliente na prestação do serviço.

- Segurança: a capacidade de transmitir confiança ao cliente, os funcionários devem apresentar conhecimento no serviço que está sendo prestado.

- Empatia: atenção individualizada que é oferecida ao cliente durante a prestação do serviço.

A qualidade do serviço será avaliada subtraindo a expectativa pela percepção, quanto maior a pontuação melhor a qualidade do serviço (OLALDE, et al. 2011). $\mathrm{Na}$ escala o cliente irá informar seu grau de concordância e discordância em relação a cada item por meio de uma escala Likert que varia de 1 a 7 pontos, sendo 1 que discorda totalmente e 7 concorda plenamente. Sendo assim, a aplicação da escala é compreendida por etapas: a primeira no qual o cliente avaliará suas expectativas em relação ao serviço, na segunda parte suas percepções e na terceira etapa será realizado uma comparação entre sua expectativa e percepção para avaliação da qualidade do serviço: Expectativa $>$ Percepção = qualidade pobre; Expectativa =Percepção =qualidade aceitável; Expectativa $<$ Percepção =qualidade boa.

Para que a qualidade no serviço seja avaliada por meio da aplicação da escala SERVQUAL é fundamental que o indivíduo tenha conhecimento claro do que é qualidade e como medi-la. É necessário também que seja realizada avaliação da qualidade a longo prazo, com o intuito de avaliar se as melhorias que foram realizadas atenderam as expectativas do cliente ao longo do tempo ou se as expectativas em relação ao serviço mudaram (ASUBONTENG et al. 1996).

\subsection{Segurança do Paciente}


A segurança do paciente bem como a melhoria na qualidade da assistência tem sido caracterizada como um movimento mundial, que envolve esforços de todo o sistema da saúde, com o intuito de promover um ambiente seguro, com redução de erros e falhas (DIAS, MEKARO et al. 2014).

A segurança do paciente se caracteriza como "a redução de danos desnecessários no cuidado à saúde". Estes danos são atualmente definidos como eventos adversos (EAs), caracterizados como complicações ou lesões não intencionais ocasionados durante a assistência prestada, podendo proporcionar incapacidade temporária ou permanente, prolongamento no tratamento e no tempo de internação e até meso a morte sem correlação com a doença que favoreceu a internação, favorecendo também a redução da confiança do usuário pelo serviço, podendo acarretar ainda em danos psicológicos (NUNES, BARROS et al. 2014).

Dentre os profissionais mais propensos a acometer eventos adversos são a equipe de enfermagem devido à realização de procedimentos invasivos e por permanecer em maior parte do tempo no cuidado ao paciente (NUNES, BARROS et al. 2014).

Em 1999 o Instituto de Medicina dos Estados Unidos realizou a publicação do relatório "Errar é Humano: construindo um sistema de saúde mais seguro", no qual foram abordados que cerca de 44.000 a 98.000 pessoas morriam a cada ano nos ambientes hospitalares em conseqüência de eventos adversos que poderiam ser evitáveis e a partir disso os movimentos para a segurança do paciente nos serviços de saúde começaram (NUNES, BARROS et al. 2014). Estima-se que em Portugal ocorrem de 1.330 a 2.900 mortes anuais devido a erros cometidos por equipe médica, representando um problema de saúde pública e ameaça à segurança do paciente (MARINHO, RUÃO et al. 2015).

A redução de eventos adversos tornou-se urgente em todo mundo, fatores como más condições de trabalho, sobrecarga, desmotivação profissional, número reduzido de profissionais, utilização de recursos tecnológicos sem pessoal qualificado, não realização da Sistematização da Assistência de Enfermagem fundamental no trabalho da equipe de enfermagem, podem favorecer a ocorrência de erros e a falta de capacitação dos profissionais de saúde com a utilização de novas tecnologias são riscos adicionais para prestação da assistência. Medidas simples podem ser adotadas de maneira a prevenir, reduzir erros e garantir a 
segurança como a implementação de protocolos específicos juntamente com educação continuada dos profissionais (OLIVEIRA et al. 2014).

$\mathrm{Na}$ assistência em enfermagem os erros e falhas mais comuns são na administração de medicamentos, na transferência de pacientes, na comunicação, na incidência de quedas, úlceras por pressão, na identificação do paciente e na ocorrência de infecção relacionada à assistência à saúde. Diante disto, a compreensão de possíveis riscos, o conhecimento teórico prático para o cuidado à saúde e das normas e políticas da instituição são fatores importantes à equipe de enfermagem para a melhoria na assistência (OLIVEIRA, LEITAO et al. 2014).

\subsection{Estratégia de melhoria para Segurança do Paciente}

Segundo a Portaria número 2616 de 12 de maio de 1998, do Ministério da Saúde, Infecção Hospitalar $(\mathrm{IH})$ é aquela adquirida após a admissão do paciente no ambiente hospitalar e que pode se manifestar durante a internação ou após a alta, quando esta estiver relacionada com a internação ou a procedimentos que foram realizados em âmbito hospitalar (WATANABE, ALMEIDA et al. 2014).

A Infecção Hospitalar (IH) muitas das vezes se torna inevitável devido ao ambiente em que o paciente está exposto, tornando-o mais suscetível para o desenvolvimento de processos infecciosos causados por microorganismos. No Brasil as IH são consideradas um problema de saúde pública, pois, elevam os custos no tratamento, aumentam os dias de internação podendo ocasionar a morte (DUTRA, COSTA et al. 2015), sendo que de 5 a 10\% dos pacientes admitidos em hospitais adquirem infecção hospitalar (ABELA e BORG, 2012).

As IH são consideradas mais graves em pacientes internados em Unidade de Terapia Intensiva (UTI) devido à sua gravidade clínica e realização de muitos procedimentos invasivos como realização de cateter venoso central, ventilação mecânica e passagem de sonda vesical de demora, relacionado também ao maior período de tempo de internação do paciente, à prescrição de antimicrobianos e ao próprio ambiente que favorece a proliferação de microorganismos. Os pacientes institucionalizados na UTI abrangem um pequeno grupo do ambiente hospitalar representando 5 a $10 \%$ do total, porém, representam um risco maior de adquirir infecção hospitalar de 5 a 10 vezes mais do que em outro setor hospitalar (PERNA, PUIATTI et al. 2015).

Além disto, as infecções ocasionadas por patógenos multirresistentes representam um grande desafio na saúde pública, pois, a resistência bacteriana 
ocasionada pela perda da eficácia de antibióticos, associados ainda ao baixo desenvolvimento e descoberta de novos antibióticos leva a chamada "crise de resistência bacteriana", sendo importante que estratégias de vigilância para detecção precoce de infecção ocorra para reduzir tempo de exposição dos pacientes aos micrrorganismos (LEBLEBICIOGLU, MORALES et al. 2016). Na Europa cerca de 25.000 pessoas morrem por ano em decorrência da resistência aos antibióticos (SIMÕES et al. 2016).

Uma das medidas mais simples e eficazes para que haja a redução no número de infecções hospitalares é a HM, portanto, muitas das vezes a adesão a esta prática pelos profissionais de saúde permanece abaixo do esperado (MU, XU et al. 2016). A hm tem sido preconizada como estratégia mais eficaz para redução de IH em todos os Programas de Controle de Infecção (SHOBOWALE, ADEGUNLE e ONYEDIBE, 2016).

Foram comprovados que a prática de HM realizada pela friccção com água e sabonete ou preparação alcoólica contribuem para a redução de 15 a 30\% das infecções hospitalares e ainda reduzir a resistência à Staphylococcus aureus. A falta de adesão dos profissionais de saúde a esta prática pode ser evidenciada pela falta de implementação de estratégias e de capacitação profissional (SHOBOWALE, ADEGUNLE e ONYEDIBE, 2016).

A HM é difinida como ato realizado pelo profissional de saúde com o objetivo de limpá-las, seja com a utilização de preparação alcoólica ou água e sabonete. A Organização Mundial de Saúde (OMS) preconiza que a higienização aconteça antes do contato com o paciente, antes da realização de procedimentos invasivos, após retirar as luvas, após contato com superfícies próximas ao paciente, após contato com fluidos corporais e após a mudança de um sítio contaminado para outro no mesmo paciente (OLIVEIRA e PAULA, 2011). .

A HM é considera medida primária para redução e controle de $\mathrm{H}$, porém, deve-se também levar em consideração que a rotina e sobrecarga de trabalho contribui para não cumprimento das etapas de higienização das mãos e favorece o aumento do número de oportunidades perdidas para $\mathrm{HM}$, sendo esta prática na maioria das vezes negligenciada devido a prioridade de se realizar atividades de cuidado (BATHKE, CUNICO et al. 2013).

A utilização de álcool gel para higienização das mãos tornou-se um método mais simples e rápido em relação a tradicional fricção com água e sabão e que pode 
substituir a lavagem com água e sabão desde que as mãos não estejam visivelmente sujas (SIQUEIRA, FIGUEIREDO et al. 2012). A a fricção com álcool gel é satisfatória, desde que, se realize a técnica correta e a quantidade correta do produto. Estudo demonstra que a quantidade ideal de álcool gel para higienização é de $2 \mathrm{ml}$ com 30 segundos de duração da técnica e já para fricção com água e sabonete a duração será de 40 a 60 segundos (JI e JEONG, 2013).

Umas das medidas denominadas como padrão-ouro para analisar a aderência dos profissionais de saúde à $\mathrm{HM}$ é a observação direta, que se caracteriza pela inserção no ambiente de um trabalho de um observador para que este possa verificar a adesão dos profissionais a esta prática. A observação deve ocorrer analisando os cinco momentos primordias em que a prática é recomendada, que são: antes do contato com o paciente, antes da realização de procedimento asséptico, após riscos de contato com fluidos corporais, após o contato com o paciente e após o contato com superfícies próximas ao paciente. Estes momentos são classificados como de alto risco para transmissão de microorganismos, por isso a necessidade de se realizar a prática de higienização de acordo com estes cinco momentos (OLIVEIRA e PAULA, 2011).

Estratégias devem ser implementadas a fim de promover uma maior adesão de HM pelos profissionais, tais como estabelecimento de planos e metas e o envolvimento dos líderes e equipe (BATHKE, CUNICO et al. 2013). A educação permanente em saúde vem sendo desenvolvida pelos serviços de saúde, constituindo-se de uma estratégia fundamental que tem como finalidade obter maior segurança para a realização dos cuidados à saúde com profissionais mais qualificados (SILVA et al. 2014).

\section{CONSIDERAÇÕES FINAIS}

A qualidade nos serviços, principalmente no âmbito da saúde, constitui-se de fator fundamental para que se obtenha uma qualidade na assistência. O controle de infecção hospitalar é de suma importância para que se obtenha esta qualidade, visto que, uma das medidas mais simples e eficazes para prevenção de infecções hospitalares é por meio da prática de higienização das mãos pelos profissionais de saúde. Nas instituições de saúde é fundamental que estratégias sejam implementadas a fim de promover uma maior aderência à higiene das mãos com o 
estabelecimento de planos e metas por parte dos gestores. A educação permanente dos profissionais também constitui-se de peça chave para obter segurança e qualidade na assistência, devido a uma maior qualificação da equipe.

\title{
The Hospital Infection Control as an indicator for Quality in Health Care
}

\begin{abstract}
The quality of services is directly related to the ability to meet customer needs. The movement for quality have expanded to the health sector and hospital infection control is one of the measures to evaluate the quality of care because such control allows the health institution to assess the structure in which the service is provided, as is It is carried out and the results of such assistance, evidenced by the increase or decrease in infections related to health. The evaluation of the practice of hand hygiene of health professionals is one of the most important steps to verify adherence to the practice by professionals because the IRAS are a large majority of the causes of morbidity and mortality in people who undergo medical procedures, and hand of health workers are on the main route of transmission of microorganisms.
\end{abstract}

Keywords: nosocomial infection; quality control; organizational culture

\section{Referências}

ABELA, N.; BORG, M. A. Impact on hand hygiene compliance following migration to a new hospital with improved resources and the sequential introduction ofWorld Health Organization recommendations. American Journal of Infection Control, 40: 737-41, 2012. Disponível em: <http://www.ncbi.nlm.nih.gov/pubmed/22285712> Acesso em 17 de novembro de 2014.

AGHAMOLAEI, T.; EFTEKHAARI, T. E.; RAFATI, S.; KAHNOUJI, K.; AHANGARI, S.; SHAHRZAD, M.E. et al. Service quality assessment of a referral hospital in Southern Iran with SERVQUAL technique: patients' perspective. BMC Health Services Research, $14: 322, \quad 2014 \quad$ Disponível em: < http://bmchealthservres.biomedcentral.com/articles/10.1186/1472-6963-14322 > Acesso em 15 de junho de 2016. 
ALBUQUERQUE, J.D.; COSTA, M.B.S.; SALAZAR, P.E.L. Avaliação da Qualidade do Gerenciamento Hospitalar na Percepção dos Profissionais. Revista Brasileira de Ciências da Saúde, 16 (2) Páginas 205-212 2012. Disponível em: < http://periodicos.ufpb.br/ojs/index.php/rbcs/article/view/12620/7309 > Acesso em 15 de julho de 2015.

ALVES, D.F.S.; GUIRARDELLO, E.B. Ambiente de trabalho da enfermagem, segurança do paciente e qualidade do cuidado em hospital pediátrico. Rev Gaúcha Enferm, 37(2); 2016. Disponível em: < DOI: http://dx.doi.org/10.1590/19831447.2016.02.58817> Acesso em 17 de outubro de 2016.

ASEFZADEH, S.; GHOLAMI, S.; RAJAEE, R.; NAFAJI, M.; ALIJANZADEH, M. Evaluation of the Quality of Health Service Providers: The Iranian People Perspective 2014. Electronic Physician, 8(3):2073-2080, 2016. Disponível em: <DOI:10.19082/2073> Acesso em 20 de agosto de 2016.

ASUBONTENG, P.; MCCLEARY, K.J.; SWAN, J.E. SERVQUAL revisited: a critical review of service quality. Journal of Services Marketing, (10)6;62 - 81, 1996. Disponível em: < http://dx.doi.org/10.1108/08876049610148602> Acesso em 15 de julho de 2016.

BABAKUS, E.; MONGOLD, G. Adapting the SERVQUAL Scale to Hospital Services: An Empirical Investlgatlon. Health Services Research, 26:6, 1992. Disponível em: < http://www.ncbi.nlm.nih.gov/pmc/articles/PMC1069855/pdf/hsresearch000750070.pdf $>$ Acesso em 15 de junho de 2016.

BATHKE, J.; CUNICO, P. A. et al. INFRAESTRUTURA E ADESÃO À HIGIENIZAÇÃ̃O DAS MÃOS: DESAFIOS À SEGURANÇA DO PACIENTE. Rev Gaúcha Enferm ;34(2):78-85, 2013. Disponível em: <http://www.scielo.br/scielo.php?pid=S198314472013000200010\&script=sci_arttext> Acesso em: 20 de novembro de 2014.

DIAS, J. D.; MEKARO, K.S.; TIBES, C.M.S.; MASCARENHAS, S.H.Z. COMPREENSÃ̃O DE ENFERMEIROS SOBRE SEGURANÇA DO PACIENTE E ERROS DE MEDICAÇÃO. Rev Min Enferm. out/dez; 18(4): 866-873, 2014. Disponível em: < http://www.reme.org.br/artigo/detalhes/969 > Acesso em 20 de dezembro de 2015.

DUTRA, G. G.; COSTA, M. P. et al. Nosocomial infection control: role of the nurse. Nosocomial infection control. J. res.: fundam. care. online 7(1):2159-2168.jan./mar. 2015. Disponível em: < http://www.seer.unirio.br/index.php/cuidadofundamental/article/view/3571/pdf $1470>$ Acesso em 01 de agosto de 2015.

JI, Y.J.; JEONG, J.S. Comparison of Antimicrobial Effect of Alcohol Gel according to the Amount and Drying Time in Health Personnel Hand Hygiene. J Korean Acad 
Nurs. Jun;43(3):305-311, 2013. Disponível em: < http://synapse.koreamed.org/search.php?where=aview\&id=10.4040/jkan.2013.43.3.3 05\&ode=0006JKAN\&vmode=PUBREADERhttp://synapse.koreamed.org/search.php? where=aview \&id=10.4040/jkan.2013.43.3.305\&code=0006JKAN\&vmode=PUBREAD ER>. Acesso em 10 de junho de 2016.

LEBLEBICIOGLU, H.; MORALES, A.J.R; ROSSOLINI, G.M.; LEZ, R.L.; ZAHAR, J.R.; RELLO, J. Management of infections in critically ill returning travellers in the intensive care unit-l: considerations on infection control and transmission of resistance. Int J Infect Dis., Jul;48:113-7, 2016. Disponível em: < http://www.ncbi.nlm.nih.gov/pubmed/27134158 > Acesso em 14 de janeiro de 2016.

MARINHO, S.; RUÃO, T.; LOPES, F.; FERNANDES, L. O erro médico na imprensa portuguesa: quando os pacientes fazem parte da notícia. Saúde Soc. São Paulo, v.24, n.4, p.1362-1376, 2015. Disponível em: < http://www.scielo.br/pdf/sausoc/v24n4/1984-0470-sausoc-24-04-01362.pdf > Acesso em 15 de julho de 2016.

MASSOCO, E. C.P.; MELLEIRO, M.M. COMUNICATION AND PATIENT SAFETY: PERCEPTION OF THE NURSING STAFF OF A TEACHING HOSPITAL. Rev Min Enferm. abr/jun; 19(2): 192-195, 2015. Disponível em: < DOI: 10.5935/14152762.20150034> Acesso em 15 de janeiro de 2016.

MU, X.; XU, Y.; YANG, T.; ZHANG, J.; WANG, C.; LIU, W.; CHEN, J.; TANG, L.; YANG, $H$. Improving hand hygiene compliance amonghealthcare workers: an intervention study in aHospital in Guizhou Province, China. Braz J Infect Dis. 2016; 1413-8670, 2016. Disponível em: < http://www.ncbi.nlm.nih.gov/pubmed/27351752 > Acesso em 10 de junho de 2016.

NUNES, F.D.O.; BARROS, L.A.; AZEVEDO, R.M.; PAIVA, S.S. Segurança do paciente: como a enfermagem vem contribuindo para a questão? J. res.: fundam. care. Online, abr./jun. 6(2):841-847, 2014. Disponível em: < http://www.seer.unirio.br/index.php/cuidadofundamental/article/viewFile/3007/pdf 12 $\underline{97}>$ Acesso em 20 de novembro de 2015.

OLIAEE, Z.; JABBARI, A.; EHSANPOUR, S. An investigation on the quality of midwifery services from the viewpoint of the clients in Isfahan through SERVQUAL model. Oliaee Z, Jabbari A, Ehsanpour S. An investigation on the quality of midwifery services from the viewpoint of the clients in Isfahan through SERVQUAL model. Iranian Journal of Nursing and Midwifery Research; 21(3):291-296, 2016. Disponível em: < http://www.ncbi.nlm.nih.gov/pmc/articles/PMC4857664/\#!po=3.12500 > Acesso em 07 de junho de 2016.

OLALDE, M.G.C.; SAWADA, N. O.; HAYASHIDA, M.; MENDES, I.A.C.; TREVIZAN, M.A.; GODOY, S. ESCALA SERVQUAL: VALIDACIÓN EN POBLACIÓN MEXICANA. Texto Contexto Enferm, Florianópolis, Jul-Set; 20(3): 526-33, 2011. Disponível em: 
$<$ http://www.scielo.br/scielo.php?script=sci arttext\&pid=S010407072011000300014 $>$ Acesso em 20 de junho de 2016.

OLIVEIRA, A. C.; PAULA, A. O. Monitoração da adesão à higienização das mãos: uma revisão de literatura. Acta Paul Enferm, 24(3):407-13, 2011. Disponível em: <http://www.scielo.br/scielo.php?script=sci_arttext\&pid=S010321002011000300016> Acesso em 17 de novembro de 2014.

OLIVEIRA, J.L.C.; PAPA, M.A.F.; WISNIEWSKI, D.; INOUE, K.C.; COSTA, M.A.R.; MATSUDA, L.M. QUALIDADE DO CUIDADO: CONCEPÇÕES DE GRADUANDOS DE ENFERMAGEM. Rev Min Enferm. jan/mar; 19(1): 29-35, 2015. Disponível em: < DOI: 10.5935/1415-2762.20150003 015> Acesso em 15 de janeiro de 2016.

OLIVEIRA, R.M.; LEITÃO, I.M.T.A.; SILVA, L.M.S.; FIGUEIREDO, S.V.; SAMPAIO, R.L. Estratégias para promover segurança do paciente: da identificação dos riscos às práticas baseadas em evidências. Esc Anna Nery; 18(1):122-129, 2014. Disponível em: < http://www.scielo.br/scielo.php?script=sci arttext\&pid=S141481452014000100122 > Acesso em 16 de outubro de 2015.

PADILHA, E.F.; MATSUDA, L.M. Qualidade dos cuidados de enfermagem em terapia intensiva: avaliação por meio de auditoria operacional. Rev Bras Enferm, jul-ago; 64(4): 684-91, 2011. Disponível em: $<$ http://www.scielo.br/scielo.php?script=sci arttext\&pid=S0034$\underline{71672011000400009}$ > Acesso em 10 de janeiro de 2016.

PARASURAMAN, A.; ZEITHAML, V.A.; BERRY, L.L. SERVQUAL: A MULTIPLE-ITEM SCALE FOR MEASURING CONSUMER PERCEPTIONS OF SERVICE QUALITY. Journal of Retailing; 64(1): 12-40, 1998. Disponível em: < http://areas.kenanflagler.unc.edu/Marketing/FacultyStaff/zeithaml/Selected\%20Public ations/SERVQUAL\%20A\%20Multipleltem\%20Scale\%20for\%20Measuring\%20Consu mer\%20Perceptions\%20of\%20Service\%20Quality.pdf> Acesso em 12 de junho de 2015.

PERNA, T.D.G.S.; PUIATTI, M.A.; PERNA, D.H.; PEREIRA, M.M.; COURI, M.G.; FERREIRA, C.M.D. Prevalência de infecção hospitalar pela bactéria do gênero klebsiellaem uma Unidade de Terapia Intensiva. Rev Soc Bras Clin Med; abrjun;13(2):119-23, 2015. Disponível em: < http://files.bvs.br/upload/S/16791010/2015/v13n2/a4740.pdf $>$ Acesso em 12 de junho de 2016.

SCHIESARI, L.M.C. Avaliação externa de organizações hospitalares no Brasil: podemos fazer diferente? Ciência \& Saúde Coletiva; 19(10):4229-4234, 2014. Disponível em: < DOI: 10.1590/1413-812320141910.21642013 > Acesso em 15 de julho de 2015.

SHOBOWALE, E.O.; ADEGUNLE, B.; ONYEDIBE, K. . An assessment of hand hygiene practices of healthcare workers of a semi-urban teaching hospital using the five moments of hand hygiene. Nigerian Medical Journal: Journal of the Nigeria Medical Association;57(3):150-154, 2016. Disponível em: < http://www.ncbi.nlm.nih.gov/pmc/articles/PMC4924395/?report=reader> Acesso em 16 de junho de 2016. 
SIQUEIRA, S. L.; FIGUEIREDO, A. E; FIGUEIREDO, C. E. P.; D'AVILA, D. O.

Comparação entre duas técnicas de higienização das mãos em pacientes de diálise peritoneal. J Bras Nefrol ;34(4):355-36, 2012. Disponível em: < http://www.scielo.br/pdf/ibn/v34n4/v34n4a08.pdf> Acesso em 11 de maio de 2016.

SILVA. C. T.; TERRA, M.G.; CAMPONOGARA, S.; KRUSE, M.H.L.; ROSO, C.C.;

XAVIER, M.S. Educação permanente em saúde a partir de profissionais de uma residência multidisciplinar: estudo de caso. Rev Gaúcha Enferm, set;35(3):49-54, 2014 Disponível em:

http://www.scielo.br/scielo.php?pid=S1983-

$14472014000300049 \&$ script=sci arttext\&tIng=pt> Acesso em 11 de maio de 2016.

SIMÕES, A.S.; COUTO, I.; TOSCANO, C. GONÇALVES, E.; PÓVOA, P.; VIVEIROS, M.; LAPÃO, L.V. Prevention and Control of Antimicrobial Resistant Healthcare- Associated Infections: The Microbiology Laboratory Rocks! Frontiers in Microbiology. 7:855, 2016. Disponível em: <Erro! A referência de hiperlink não é válida.> Acesso em 10 de junho de 2016.

SOUZA, E. S.; BELEI, R. A. et al. MORTALIDADE E RISCOS ASSOCIADOS A INFECÇÃO RELACIONADA À ASSISTÊNCIA À SAÚDE. Texto Contexto Enferm, Jan-Mar; 24(1): 220-8, 2015. Disponível em:

$<$ http://www.scielo.br/pdf/tce/v24n1/pt 0104-0707-tce-24-01-00220.pdf > Acesso em 01 de agosto de 2015.

URBANETTO, J. S.; GERHARDT, L.M. SEGURANÇA DO PACIENTE NA TRÍADE ASSISTÊNCIA ENSINO PESQUISA. Rev Gaúcha Enferm;34(3):8-9, 2013. Disponível em: <http://seer.ufrgs.br/RevistaGauchadeEnfermagem/article/view/43294 > Acesso em 20 de julho de 2016.

VITURI, D.W.; ÉVORA, Y.D.M. Gestão da Qualidade Total e enfermagem hospitalar: uma revisão integrativa de literatura. Rev Bras Enferm. setout;68(5):945, 2015. Disponível em: < http://www.scielo.br/pdf/reben/v68n5/00347167-reben-68-05- 0945.pdf $>$ Acesso em 12 de janeiro de 2016.

WATANABE, E.M.; ALMEIDA, V.F.; OTTUNES, A.F.; DESSUNTI, E.M.; PIERI, F.M.; MARTINS, E.A.P.; KERBAUY, G. Impacto das infecções relacionadas à assistência à saúde em pacientes acometidos por trauma. Ciências Biológicas e da Saúde, Londrina,; 36 (1); 89-98, 2015. Disponível em: < DOI: 10.5433/1679-0367.2014v35n2p89 > Acesso em 10 de maio de 2016. 